times. Most would like the appointment to last between 20-30 min. A diabetic nurse run clinic was preferred by $48.4 \%(51.6 \%$ did not want it). The best age to be transferred was between 1619 yrs.

Lessons learnt Patient input and experience can give useful insight when designing a model for transition for young people with diabetes.

Message for others Patient views are imperative to bring about a change in the service made for them.

\section{G586(P) PHOTOTHERAPY MANAGEMENT IN JAUNDICED BABIES: JAUNDICE MANAGEMENT TOOL}

JL McDermott. The Neonatal Intensive Care Unit, Queen Alexandra Hospital, Portsmouth, UK

\subsection{6/archdischild-2015-308599.535}

Context This quality improvement work aimed to improve the management of infants on the postnatal ward (PNW) at Queen Alexandra Hospital, Portsmouth who were undergoing phototherapy for the management of neonatal jaundice. The main staff groups targeted were those involved in the care of infants on the PNW (neonatal trainees, ANNPs, midwives and health care support workers).

Problem In 2010, the National institute for Health and Clinical Excellence (NICE) published evidence-based guidelines aimed at standardising the management of neonatal jaundice across the UK. In 2014, an audit of the management of jaundiced infants on the PNW against NICE standards revealed deficiencies relating to the timing of repeat serum bilirubin measurement (SBR) with only $21 \%$ having a repeat SBR which complied with the NICE standard. This in turn was potentially putting babies at risk from jaundice.

Assessment of problem and analysis of its causes The NICE phototherapy pathway was displayed on a wall on the PNW. Its complex nature meant that many members of staff did not use it to guide their management of infants under phototherapy. Midwives would often ask the neonatal trainees for advice regarding management and timings of future SBR samples. With trainees changing frequently and a variety of level of experience, there appeared to be a lack of consistency in the management of neonatal jaundice.

Intervention A Jaundice Management Tool (JMT) based on the NICE guidance was designed to use alongside the NICE jaundice graphs (see figure 1). This formally documents times of SBRs, guides the phototherapy management, and advises on timings of subsequent SBRs.

Study design A reaudit following a month of using the JMT will investigate whether adherence to NICE guidelines is improved.

Strategy for change The JMT was printed, attached to the jaundice treatment threshold graphs and distributed to all the PNW. At the same time, midwives, healthcare support workers and neonatal trainees underwent training in the use of the JMT.

Measurement of improvement Following a month of using the JMT on the postnatal ward, a formal reaudit will be underway, along with a questionnaire to provide feedback regarding the JMT, and assess how user-friendly it is.

Effects of changes So far the JMT has resulted in improved clinical care with more babies having appropriately timed repeat SBRs. In addition, it appears that the JMT has removed confusion around the timing of repeat SBRs and has encouraged a standardised approach across disciplines who are involved in the care of postnatal ward babies.

Lessons learnt I have learnt that in order to implement the JMT, it was crucial that those involved were engaged in the process and keen to be part of the change in practice. It necessitated me liaising with a multidisciplinary group and I learnt to change my style depending on the disciplines I was addressing.

Message for others In line with several studies relating to practice change, passive dissemination of guidelines is rarely effective. It is not sufficient to merely display guidance but necessitates meeting with the various disciplines and educating them on the need for change and how it will improve patient care

The active dissemination strategy using a multifaceted used in this project seemed effective in improving practice. Useful elements included tailoring the implementation of change to the

\begin{tabular}{|c|c|}
\hline $\begin{array}{l}\text { Name } \\
\text { Date of Birth } \\
\text { Q number } \\
\text { (attach addressograph if available) }\end{array}$ & $\begin{array}{l}\text { If a baby looks ja undiced, perform an SBR or trans cutaneous bilirubin measurement. If result plots } \\
\text { above treatment line, start phototherapy and see below: } \\
\text { 1. Start multiple phototherapy if the SBR is rising rapidly (>8.5micromol/ } / / \mathrm{hr} \text { ) or within } 5 \text { boxes } \\
\text { below the threshold for exchange transfusion after } 72 \text { hours - INFORM CON SULTANT INVOLIVED } \\
\text { 2. Perform FBC, blood group and DAT in all babies treated with phototherapy (heel prick) } \\
\text { 3. Consider other investigations e.g. septic screen investigations depending on the clinical situation } \\
\text { - START Photother apy } \rightarrow \text { Repeat SBR in 4-6 hours }\end{array}$ \\
\hline & - STABLE / FALLING SBR $\rightarrow$ Repea SBR every $8 \mathrm{hrs}$ \\
\hline Time of birth & $\begin{array}{l}\text { - STOP phototherapy if SBR greater than or equal to } 5 \text { boxes below tr eatment line } \\
\text { - CHECK SBR } 12 \text { hours after turning off lights (rebound SBR) }\end{array}$ \\
\hline
\end{tabular}

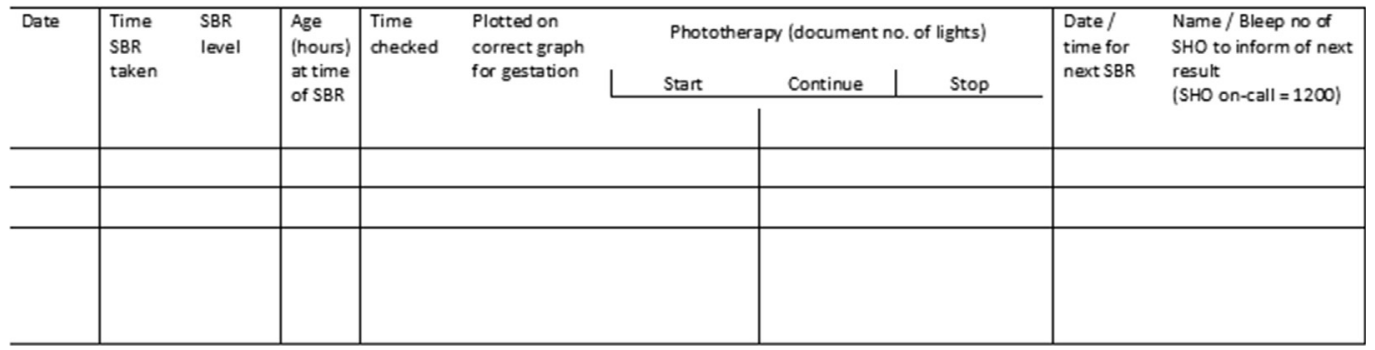


local setting and users, education of staff groups, and the JMT itself as both a reminder and an easily accessible guide to care.

Charts and images: Please see Figure 1: Jaundice Management Tool

\section{G587(P) IMPROVING UNDERSTANDING OF CHILDREN ATTENDING PAEDIATRIC OUTPATIENTS}

X Poblete, F Morrison, N Prevatt, C Jennings. Community Paediatrics, Northwest London Hospitals Trust, London, UK

\subsection{6/archdischild-2015-308599.536}

Context A busy paediatric outpatient department in a UK district general hospital

Problem There is currently no information provided to children by the trust. This may have been adversely affecting our paediatric outpatients. By providing an information leaflet to children we hoped to improve their understanding and patient experience.

Assessment of problem and analysis of its causes Children are often overlooked in relation to patient information. Paediatric leaflets are usually written for parents. We surmised that children would benefit from understanding why they were coming to outpatients and what they could expect to happen while they were there. When children are more informed they may be more relaxed and therefore more cooperative with the physical examination. Children who have a positive experience of their first outpatient appointment may be more comfortable with future appointments.

Intervention A trust patient information leaflet that parents could go through with their children prior to attending their first paediatric outpatient appointment.

Study design and strategy for change Send out information leaflet with all new appointments for children aged 2-12 years (over an 8 month period) with a request that parents go through it with their child before attending the appointment. Leaflet designed with assistance of senior paediatric speech and language therapist to ensure that age-appropriate language used. Pictures were included to illustrate components of outpatient

\section{Of those who received the leaflet: Did you find it useful?}

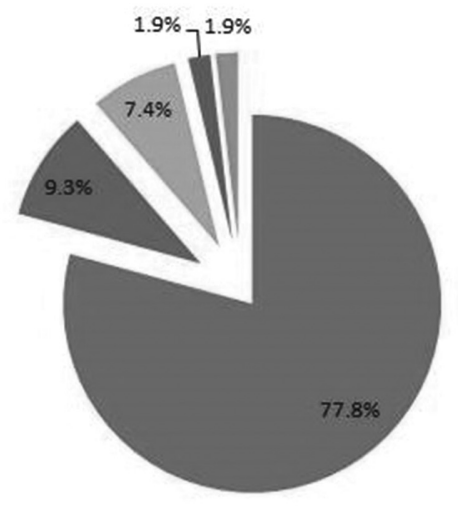

appointment, such as being weighed by nurse. Leaflet approved by in-hospital communications department.

Measurement of improvement A questionnaire given to parents in outpatient reception. Parents were asked whether they received leaflet, whether they went through it with their child and whether they felt it was helpful. If they didn't receive it they were asked whether they thought it may have been helpful.

We also asked our clinicians to ask the children pre-defined questions prior to beginning the consultation to try and capture the children's views verbatim. Most clinicians did not complete these questions. This may reflect time pressure during appointments.

We collated the questionnaire responses and analysed the data. We also obtained further suggestions for improvement from parents.

Key findings:

$42 \%$ of parents who had not received the leaflet reported that it would have been helpful.

However $78 \%$ of parents who received the leaflet prior to the appointment reported that it was useful (Figure 1).

When asked to explain what their child understood about why they were coming to clinic and what would happen $24 \%$ left the question blank, possibly due to a language barrier.

Some comments from parents who had not received leaflet prior to appointment included 'I wish we could have received this beforehand to go through with my child' and 'it's always important to communicate with children and explain to them everything in advance. This will help to keep them calm and happy.'

A parent who had received leaflet commented 'perhaps a child-friendly version - in colour and larger font would be helpful'.

Effects of changes Our work demonstrated that most parents in our population valued the provision of this information to their children. We used parents' views as a proxy for their children's which may not be accurate. Over time, as more leaflets are distributed, it may be possible to investigate whether children who received the leaflet had a more positive experience of outpatients than those who didn't.

\section{Of those who did not receive the leaflet: Do you feel it would have been useful?}

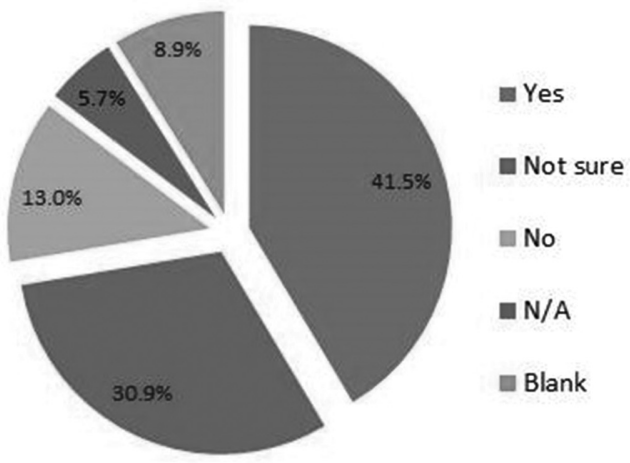

